\title{
Lower Body Lift: Manejo integral del tronco inferior en pacientes con baja masiva de peso. Serie de casos y descripción de la técnica quirúrgica
}

'Hospital Clínico de la Universidad de Chile, Santiago, Chile.

Recibido el 11 de septiembre de 2017 y aceptado para publicación el 18 de noviembre de 2017

Correspondencia a: Dr. Stefan Danilla E. drstefandanilla@gmail.com

\author{
Stefan Danilla E. ${ }^{1}$, Carlos Domínguez C. ${ }^{1}$, José Tomas Ganz V. ${ }^{1}$, \\ Ekaterina Troncoso O. ${ }^{1}$, Marco Ríos V. ${ }^{1}$, Patricio Andrades C. ${ }^{1}$, \\ Juan Pablo Cisternas V. ${ }^{1}$, Cristian Erazo C. ${ }^{1}$ y Sergio Sepúlveda P. ${ }^{1}$
}

Lower Body Lift: Integral management of the lower trunk in patients with massive weight loss. Case series and description of the Surgical Technique

Introduction: Obesity is a public health problem; bariatric surgery is an effective treatment to weight loss. Skin surplus and adiposity can produce physical and psychological problems. The management of these patients requires large cutaneous resections. The Lower Body Lift is an integral strategy that allows to handle abdominal, gluteal and proximal thigh area. Objective: Present a series of patients with massive weight loss using the Lower Body Lift and expose the surgical technique. Materials and Method: Cohort of patients managed with Lower Body Lift by plastic surgeons of a university clinical hospital, between January 2013 and June 2016. A descriptive analysis of the series was realized. And the surgical technique is exposed. Results: Of a total of 97 lower body contour procedures in patients with massive weight loss, $17(17.52 \%)$ were Lower Body Lift. $14(82.35 \%)$ were women, with a mean age of $39.2 \pm 11.2$ years. On average, the patients had a weight loss of $41.4 \pm 14.9 \mathrm{~kg}$, with the BMI at the time of the intervention of $26.02 \pm 6.17 \mathrm{~kg} / \mathrm{m}^{2}$. Conclusions: Patients with massive weight loss have a large cutaneous surplus that may require extensive resections to achieve satisfactory contour correction. Our patients had a weight loss of about $40 \mathrm{Kg}$ prior to surgery, reaching almost normal BMI. The Lower Body Lift technique successfully corrects the aftermath of massive weight loss, with associated low complications.

Key words: abdominoplasty; weight loss; plastic surgery.

\section{Resumen}

Introducción: La obesidad es un problema de salud pública, la cirugía bariátrica es una opción terapéutica cada vez más utilizada, facilitando bajas significativas de peso. El excedente cutáneo y adiposidad pueden producir problemas tanto físicos como psicológicos. El tratamiento de estos pacientes requiere en algunos casos de grandes resecciones cutáneas, el Lower Body Lift es una estrategia combinada que permite tratar de forma integral el área abdominal, glútea y muslo proximal. Objetivo: Presentar una serie de pacientes con pérdida masiva de peso con la técnica del Lower Body Lift y exponer la técnica quirúrgica. Materiales y Método: Cohorte de pacientes tratados con Lower Body Lift por un equipo de cirugía plástica del hospital clínico universitario, entre enero de 2013 y junio de 2016. Se realizó un análisis descriptivo de las variables analizadas y se presenta la técnica quirúrgica. Resultados: De un total de 97 procedimientos de contorno corporal inferior en pacientes con pérdida masiva de peso, 17 (17,52\%) fueron Lower Body Lift, de los cuales $14(82,35 \%)$ correspondieron a mujeres, con una edad promedio de 39,2 $\pm 11,2$ años. En promedio los pacientes tuvieron una pérdida de peso de $41,4 \pm 14,9 \mathrm{Kg}$, siendo el IMC al momento de la intervención de 26,02 $\pm 6,17 \mathrm{Kg} / \mathrm{m}^{2}$. Conclusiones: Los pacientes con pérdida masiva de peso presentan un gran excedente cutáneo que puede requerir resecciones extensas para lograr la corrección satisfactoria del contorno. Nuestros pacientes tuvieron una pérdida de peso de alrededor de $40 \mathrm{Kg}$ previo a la cirugía, alcanzando IMC casi normales. La técnica de Lower Body Lift permite corregir satisfactoriamente las secuelas de la baja masiva de peso, con bajas complicaciones asociadas.

Palabras clave: abdominoplastía; pérdida de peso; cirugía plástica. 


\section{Introducción}

La obesidad se ha consolidado como un problema de salud pública debido a su alta prevalencia, comorbilidades asociadas e impacto negativo en la calidad y expectativa de vida ${ }^{1-3}$.

A pesar de los múltiples beneficios atribuidos a pérdida masiva de peso $^{4}$, los pacientes suelen cursar con secuelas en el contorno corporal ${ }^{5}$. Producto del excedente cutáneo, laxitud de tejidos y alteración en la distribución del tejido adiposo, que se generan asociados a la pérdida masiva y rápida de peso en este tipo de pacientes ${ }^{5}$. Estas alteraciones suelen producir desconformidad con el aspecto físico, dificultando la movilidad, alterando la cicatrización de heridas, generando dermatitis e intertrigo en zonas de pliegues con el consiguiente compromiso de la higiene de los pacientes ${ }^{6-8}$. Estas alteraciones suelen llevar a un deterioro en la calidad de vida e imagen corporal pese a la baja de peso $^{9-12}$.

En el tronco medio inferior se genera un excedente cutáneo que se extiende circunferencialmente desde los flancos a la región lumbosacra. La región glútea presenta ptosis significativa, que oculta a menudo el pliegue glúteo ${ }^{13,14}$. En este grupo de pacientes, procedimientos como la abdominoplastía tradicional son insuficientes para restituir el contorno corporal en forma adecuada. Procedimientos más extensos como la abdominoplastía extendida, incluso circunferencial, no logran dar un resultado adecuado, debido al gran excedente de cutáneo y redistribución grasa, siendo necesario la realización de procedimientos más complejos, que combinan diferentes herramientas reconstructivas para poder dar una solución integral al contorno corporal del tronco inferior ${ }^{15-17}$. El Lower Body Lift es un procedimiento que combina en un tiempo quirúrgico, una abdominoplastía circunferencial con decolamiento intermitente de muslos asociado a un autoaumento glúteo, permitiendo el tratamiento de la región abdominal una elevación de la cara lateral del muslo y una mejora de la proyección y contorno glúteo ${ }^{16,18}$.

$\mathrm{Si}$ bien existen reportes a nivel internacional de series de pacientes con baja masiva de peso, sometidos a cirugía de contorno corporal y en particular utilizando la técnica del Lower Body Lift en casos seleccionados, no existen referencias a nivel nacional que describan el tratamiento de este grupo de pacientes.

El presente trabajo tiene por objeto presentar una serie de pacientes con baja masiva de peso operados con esta técnica, así como describirla.

\section{Materiales y Métodos}

Serie de casos clínicos prospectiva, obtenida de la base de datos digital de pacientes con cirugía de contorno corporal (Body-QoL www.bodyqol.cl). Se incluyó a todos los pacientes sometidos al procedimiento Lower Body Lift entre el 01 enero de 2013 y 31 de junio de 2016, por el equipo de cirugía plástica de un hospital universitario. Se excluyó los procedimientos realizados en otro Centro, en más de un tiempo quirúrgico o con antecedentes incompletos.

Se evaluó variables demográficas, clínicas, técnica quirúrgica y complicaciones. Las variables continuas se describieron con promedio y desviación estándar, las variables categóricas, con frecuencia y porcentajes. Se realizó un análisis estadístico descriptivo, utilizando STATA $13^{\circledR}$.

\section{Técnica quirúrgica: Lower Body Lift}

\section{Selección de pacientes}

La utilización de la técnica quirúrgica está determinada por la valoración previa de cada uno de los pacientes, de los excesos dermograsos a resecar, las estructuras y diferentes subunidades anatómicas a restablecer y las preferencias del paciente.

Son candidatos a este procedimiento pacientes: Edad menor a 60 años, comorbilidades estabilizadas, hábito tabáquico suspendido e IMC $<30 \mathrm{Kg} / \mathrm{m}^{2}$.

\section{Marcación y fotografía preoperatoria}

Antes de iniciar el procedimiento quirúrgico se realiza la marcación y fotografía preoperatoria.

Fotografía clínica de cuerpo completo con tomas cada $45^{\circ}$ hasta completar todo el perímetro del cuerpo. Realizando esto con brazos en posición anatómica y abducidos. Se registran las mismas secuencias en el posoperatorio, al mes y a los 6 meses.

\section{Marcación}

Previo a la marcación se evalúa con paciente de pie distribución de tejido graso, laxitud de piel y excedente cutáneo.

\section{Abdominoplastía circunferencial}

Se diseña patrón de resección circunferencial delimitado por dos líneas "inferior" y "superior", buscando ocultar la cicatriz bajo la ropa interior. Para definir "línea inferior" (línea de corte) se tracciona excedente cutáneo lumbar hacia cefálico, hasta lograr adecuada elevación de cara lateral de muslo y glúteo; diseñando "línea inferior" según el nivel que se desea proyectar a la cicatriz, se repite el mismo procedimiento en línea medial, cara late- 


\section{ARTÍCULO ORIGINAL}

ral del muslo y pared abdominal. A continuación, se diseña "línea superior" (nivel tentativo de corte superior), mediante pinch test desde la base de la "línea inferior" previamente diseñada, desde la línea media lumbar hacia el flanco. En la pared abdominal anterior se continúa trazado superior paralelo a "línea inferior". Se extrapola marcación en lado contrario.

\section{Autoaumento glúteo}

En ambos glúteos por sobre nivel de "línea inferior" y se define área de preservación de tejido subcutáneo para la confección de colgajos grasos destinados a autoaumento glúteo.

\section{Decolamiento}

Se delimita zona de decolamiento discontinuo bajo el nivel de "línea inferior" en la porción externa de ambos glúteos y en los dos tercios proximales de la cara externa de los muslos. Se define, además, área de decolamiento continuo bajo el nivel de "línea inferior" en relación a autoaumento glúteo.

\section{Liposucción}

En algunos casos se realiza liposucción complementaria en forma limitada. En ambos flancos se marca zona de lipoaspiración para desgrasar el tejido celular subcutáneo, logrando un contorno más parejo, permitiendo limitar la resección, preservando fascia de Scarpa (Figura 1).
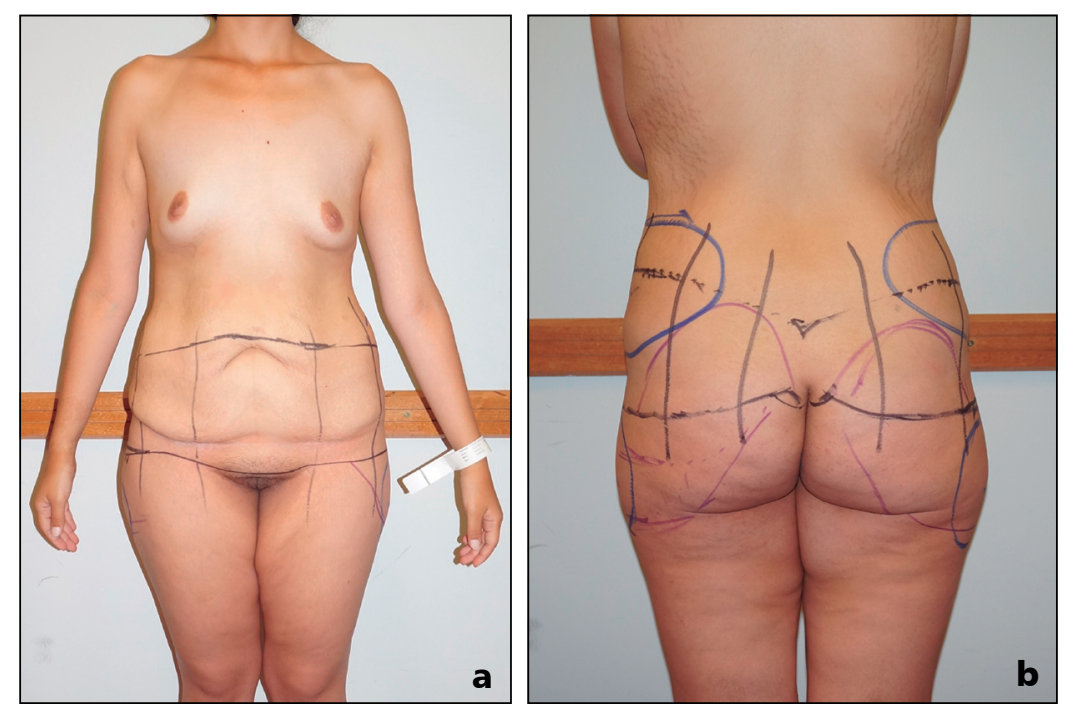

Figura 1. Marcación preoperatoria de Lower Body Lift. Paciente de sexo femenino de 31 años, con antecedente de baja de peso de 48 kg, secundaria a ByPass Gástrico. Vista anterior de marcación (a), vista posterior de marcación (b).

\section{Técnica quirúrgica}

La cirugía se inicia con paciente en decúbito prono. Incisión de piel por "línea inferior" hasta ambos flancos y se profundiza hasta fascia lumbar. Infiltración de solución vasoconstrictora $(1: 1.000 .000)$ y posterior lipoaspiración de flancos, buscando desgrasar tejido celular subcutáneo.

Decolamiento subcutáneo hacia cefálico sin sobrepasar "línea superior", tallando colgajos grasos en ambos glúteos según marcación previa y hacia lateral se profundiza el plano de resección subcutánea preservando fascia de Scarpa. En borde inferior de incisión se realiza decolamiento continuo en el espesor del tejido celular subcutáneo, asociado a decolamiento discontinuo con cánula de lipoaspiración de glúteo y muslo.

Se confecciona autoaumento glúteo con colgajo graso fijado con sutura de poliéster $2 / 0$ a fascia glútea.

Previo a completar la resección por borde superior del losanjo, se calibra la resección de excedente cutáneo, asegurando un cierre libre de tensión utilizando pinzas Pozzi. Una vez resecado se instala drenaje de presión negativa a cada lado (el cual se exteriorizará por el pubis) y se realiza cierre en tres planos con sutura barbada de reabsorción lenta, calibre $2 / 0$ subcutáneo, $3 / 0$ dermis profunda y DERMABOND $^{\text {TM }}$ PRINEO $^{\text {TM }}$ a piel.

Se continúa procedimiento en decúbito dorsal, liberando tallo umbilical, completando incisión por "línea inferior", elevación de colgajo dermograso hacia cefálico hasta identificar apéndice xifoides, procurando realizar mínimo decolamiento hacia lateral. Plicatura de rectos con sutura barbada de reabsorción lenta. Se completa abdominoplastía calibrando y resecando excedente cutáneo abdominal, sutura de piel en tres planos al igual que en región lumbar y transposición de ombligo.

\section{Principios quirúrgicos claves}

Al igual que en todos los pacientes con baja masiva de peso comprende ${ }^{19,20}$ :

- Mínimo decolamiento.

- Liposucción limitada.

- Cerrar los espacios muertos.

- Proteger hipotermia.

- Uso rutinario de drenajes.

\section{Resultados}

En el período de tiempo anteriormente referido, se llevaron a cabo un total de 1.027 procedimientos del contorno corporal en 120 pacientes con pérdida 

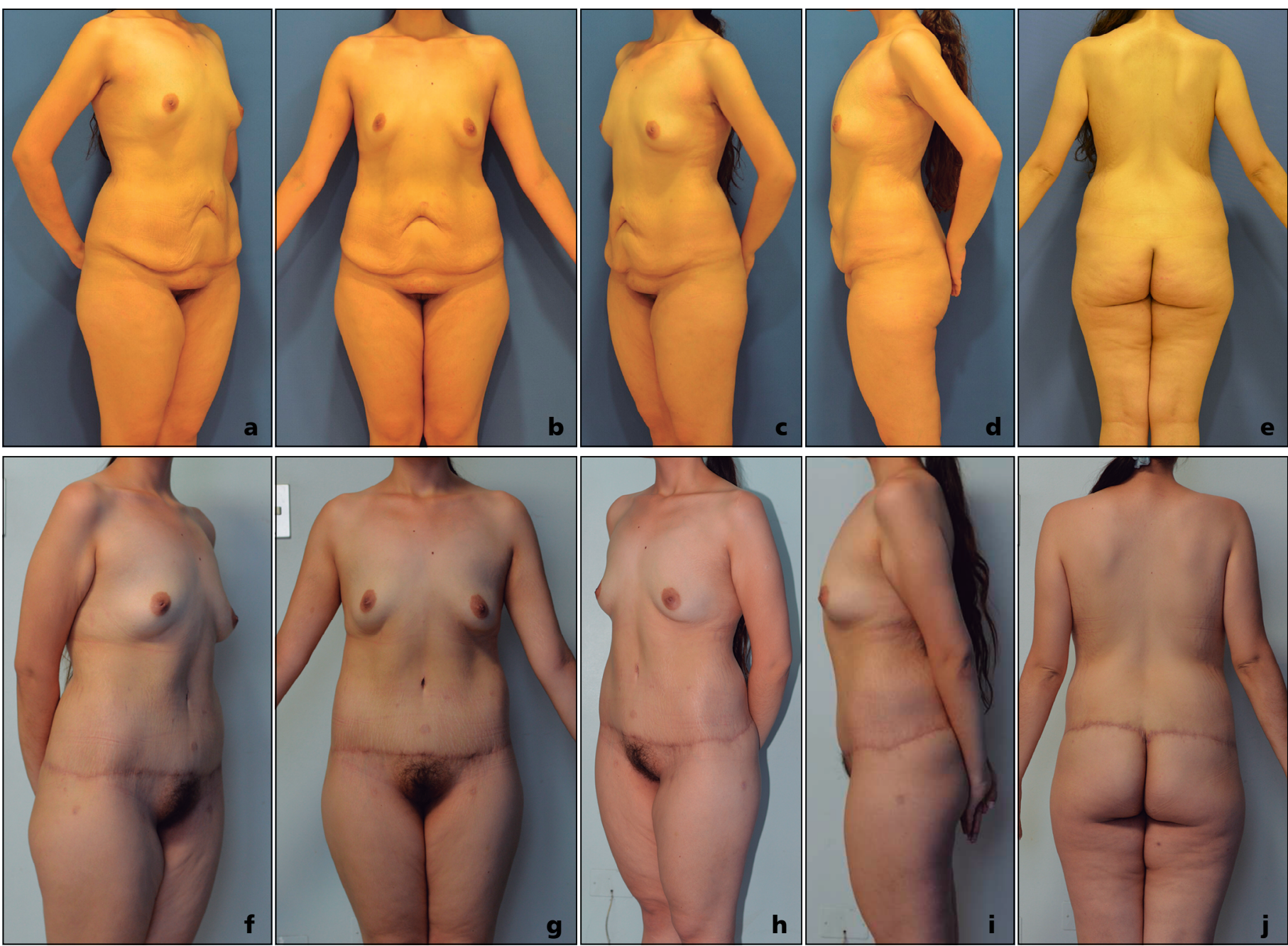

Figura 2. Serie fotográfica comparativa pre y posoperatoria de paciente de sexo femenino de 31 años. Con antecedente de baja de peso de $48 \mathrm{~kg}$, secundaria a ByPass Gástrico. Fotografías preoperatorias (a-e) y Fotografías posoperatorias (f-j)

masiva de peso. De este grupo $96(80 \%)$ pacientes se efectuaron alguna cirugía de contorno corporal del tronco inferior, siendo 17 (14,16\%) Lower Body Lift, según la técnica anteriormente expuesta. De ellos, $14(82,35 \%)$ casos correspondieron a pacientes de sexo femenino y $3(17,64 \%)$ pacientes de sexo masculino, con una edad promedio de 39,2 $\pm 11,16$ [26-53] años.

El índice de masa corporal (IMC) basal de esta serie fue $41,57 \pm 6,14 \mathrm{Kg} / \mathrm{m}^{2}$, logrando disminuir $41,48 \pm 14,92 \mathrm{Kg}$, alcanzando un IMC 26,02 $\pm 2,17$ $\mathrm{Kg} / \mathrm{m}^{2}$ previo al procedimiento. La baja masiva de peso fue alcanzada en $15(88,2 \%)$ pacientes secundario a una cirugía bariátrica, la cual fue realizada $3,15 \pm 2,23$ [1-8] años previo a la cirugía de contorno corporal.
Se utilizó liposucción como procedimiento complementario en $2(11,76 \%)$ pacientes de sexo femenino, no observándose otros procedimientos asociados en el mismo tiempo quirúrgico. El tiempo operatorio de nuestra serie correspondió a 3,92 $\pm 2,05 \mathrm{~h}$.

Seis $(35,29 \%)$ pacientes se realizaron, además, un procedimiento de contorno corporal en el tronco superior en un tiempo quirúrgico diferente, 2 $(11,76 \%)$ mastopexia sin implantes y $4(23,53 \%)$ Upper Body Lift.

La serie no registra mortalidad. Se observaron complicaciones menores en $9(52,9 \%)$ pacientes, siendo la más frecuente la dehiscencia de herida operatoria con $6(35,29 \%)$ casos, cicatriz hipertrófica en $3(17,6 \%)$ pacientes y la presencia de seroma en solo $1(5,88 \%)$ paciente. Todas estas complica- 
ciones fueron manejadas en forma conservadora, con curaciones ambulatorias.

\section{Discusión}

Al revisar la literatura, si bien existen múltiples publicaciones (series de casos, estudios de cohorte, revisiones sistemáticas y guías de recomendación) sobre cirugía de contorno corporal en pacientes con baja masiva de peso, existen pocas series destinadas en forma exclusiva a analizar pacientes con Lower Body Lift ${ }^{21-23}$.

Al igual que lo publicado por otros autores, en pacientes con secuelas cutáneas producto de la baja masiva de peso, los procedimientos de contorno corporal del tronco inferior y en particular la abdominoplastía estándar son los más realizados, siendo el Lower Body Lift reservado para pacientes con un gran excedente cutáneo; en nuestra experiencia se realizó en un $14,2 \%$ de los casos no existen otros reportes a nivel nacional, series internacionales lo describen en un $11,4-25 \%{ }^{24,25}$.

Acorde a lo reportado por otras series, estos pacientes presentan un predominio de sexo femenino, con un rango de edad entre 20 y 62 años, siendo la cirugía bariátrica el método más frecuente utilizado para bajar de peso $^{26,27}$.

Los pacientes incluidos en esta serie presentaron un menor IMC basal $\left(49,5\right.$ vs $\left.41,57 \mathrm{Kg} / \mathrm{m}^{2}\right)$ y pérdida de peso $(60,5$ vs 41,5 $\mathrm{Kg})$ en comparación con lo reportado por Kitzinger et $\mathrm{al}^{22}$, alcanzando un IMC previo a la cirugía de contorno corporal similares (27,8 y 26,0\%), el cual se encuentra bajo el límite de seguridad recomendado para procedimientos de contorno corporal ${ }^{23,28}$.

$\mathrm{Si}$ bien existen reportes de manejo del tronco superior e inferior en un tiempo quirúrgico (Total Body Lift $)^{29}$, en nuestra serie los pacientes que necesitaban un procedimiento del tronco superior, este fue realizado en un tiempo quirúrgico diferente $(35,3 \%)$, buscando disminuir las complicaciones asociadas a procedimientos muy extensos.

El desarrollo de complicaciones es un evento frecuente en este grupo de pacientes $(40-70 \%)^{22,25}$. El seroma es la complicación más frecuente reportada $(13-29 \%)^{25}$ en nuestra experiencia se presentó en un caso $(5,9 \%)$, lo cual se podría atribuir al uso rutinario de drenajes, decolamiento y liposucción limitada. La dehiscencia de herida operatoria $(35,29 \%)$ fue nuestra principal complicación, lo cual no difiere mayormente del 22-30\% de dehiscencia de herida reportada en la literatura internacional ${ }^{29,31}$. Importante resulta destacar, que la totalidad de nuestras dehiscencias fueron manejadas en forma conservadora ambulatoria con la posterior resolución ad integrum a través del cierre por segunda intención. La cicatriz hipertrófica correspondió a la segunda complicación más frecuente en nuestra experiencia $(17,6 \%)$, no observándose infección del sitio operatorio ni necrosis de piel, las cuales son complicaciones descritas por otros autores ${ }^{21,23}$.

Dada la magnitud del excedente cutáneo a resecar, comorbilidades asociadas, presencia de cicatrices abdominales previas, este grupo de pacientes representan mayor complejidad tanto en la planificación y ejecución. Si bien, el número de procedimientos necesarios para establecer rangos de seguridad en este tipo de pacientes no está planteado en la literatura, creemos que la formación de equipos quirúrgicos coordinados y experimentados, es importante en la reducido del número de complicaciones secundarias a este tipo de procedimientos.

\section{Conclusión}

La técnica del Lower Body Lift permite corregir satisfactoriamente el contorno corporal medio-bajo en este subgrupo particular de pacientes. Para ello el manejo protocolizado con una selección adecuada de pacientes, asociado al trabajo en equipo es fundamentales en el éxito de este procedimiento.

Los pacientes, en conjunto con el cirujano plástico tratante, deben estar dispuestos a hacer una compensación del riesgo de problemas menores derivados de esta técnica quirúrgica considerando la principal ventaja de este procedimiento: menos etapas en la reconstrucción del contorno corporal de la parte media-baja del cuerpo.

\section{Responsabilidades éticas}

Protección de personas y animales. Los autores declaran que para esta investigación no se han realizado experimentos en seres humanos ni en animales.

Confidencialidad de los datos. Los autores declaran que han seguido los protocolos de su centro de trabajo sobre la publicación de datos de pacientes.

Derecho a la privacidad y consentimiento informado. Los autores han obtenido el consentimiento informado de los pacientes y/o sujetos referidos en el artículo. Este documento obra en poder del autor de correspondencia.

\section{Conflicto de intereses}

Los autores declaran que no tienen conflictos de interés. 


\section{Bibliografía}

1. Vio F, Albala C, Kain J. Nutrition transition in Chile revisited: mid-term evaluation of obesity goals for the period 2000-2010. PHN 2007;11:1-8.

2. Fountaine K, Redden D, Wang C, Westfall A, Allison D. Years of Life Lost Due to Obesity. JAMA 2003;289:187-93.

3. Encuesta Nacional de Salud 2009-2010 [Internet]. 2011. Available from: http:// www.redsalud.gov.cl/portal/docs/ page/minsalcl/g_home/submenu portada 2011/ens2010.pdf

4. Lee WJ, Chong K, Lin YH, Wei JH, Chen SC. Laparoscopic Sleeve Gastrectomy Versus Single Anastomosis (Mini-) Gastric Bypass for the Treatment of Type 2 Diabetes Mellitus: 5-Year Results of a Randomized Trial and Study of Incretin Effect. OBES SURG. Springer US; 2014;24:1552-62.

5. Ellison JM, Steffen KJ, Sarwer DB. Body Contouring After Bariatric Surgery. de Zwaan M, Mitchell JE. Eur Eat Disorders Rev. 2015;23:479-87.

6. Biörserud C, Olbers T, Fagevik Olsén M. Patients' Experience of Surplus Skin After Laparoscopic Gastric Bypass. OBES SURG. 2009;21:273-7.

7. Aldaqal SM, Makhdoum AM, Turki AM, Awan BA, Samargandi OA, Jamjom H. Post-Bariatric Surgery Satisfaction and Body-Contouring Consideration after Massive Weight Loss. North American Journal of Medical Sciences 2013;5:3015.

8. Albino FP, Koltz PF, Gusenoff JA. A Comparative Analysis and Systematic Review of the Wound-Healing Milieu: Implications for Body Contouring after Massive Weight Loss. Plastic and Reconstructive Surgery 2009;124:167582.

9. Sarwer DB, Thompson JK, Mitchell JE, Rubin JP. Psychological Considerations of the Bariatric Surgery Patient Undergoing Body Contouring Surgery. Plastic and Reconstructive Surgery. 2008;121:423e-434e.

10. Mitchell JE, Crosby RD, Ertelt TW, Marino JM, Sarwer DB, Thompson JK, et al. The Desire for Body Contouring Surgery after Bariatric Surgery. OBES SURG. 2008;18:1308-12.

11. Danilla S, Cuevas P, Aedo S, Domínguez C, Jara R, Calderón ME, et al. Introducing the Body-QoL(R): A New PatientReported Outcome Instrument for Measuring Body Satisfaction-Related Quality of Life in Aesthetic and Postbariatric Body Contouring Patients. Aesthetic Plast Surg. 2016;40:19-29.

12. Song A, Rubin JP, Thomas V, Dudas J, Marra KG, Fernstrom M. Body Image and Quality of Life in Post Massive Weight Loss Body Contouring Patients. OBES SURG. 2006;14:1-11.

13. Strauch B, Herman C, Rohde C, Baum T. Mid-Body Contouring in the Post Bariatric Surgery Patient. Plastic and Reconstructive Surgery. 2006;117:220011.

14. Aly AS, Cram AE, Heddens C. Truncal body contouring surgery in the massive weight loss patient. Clinics in Plastic Surger 2004;31:611-24

15. Capella JF. Body Lift. Clinics in Plastic Surgery. 2008;35:27-51.

16. Richter DF, Stoff A, Velasco-Laguardia FJ, Reichenberger MA. Circumferential Lower Truncal Dermatolipectomy. Clinics in Plastic Surgery 2008;35:53-71.

17. Almutairi K, Gusenoff JA, Rubin JP. Body Contouring. Plastic and Reconstructive Surgery 2016;137:586e-602e.

18. Shermak MA. Body Contouring. Plastic and Reconstructive Surgery 2012;129:963e-978e.

19. Kolker AR, Lampert JA. Maximizing Aesthetics and Safety in CircumferentialIncision Lower Body Lift With Selective Undermining and Liposuction. Annals of Plastic Surgery 2009;62:544-8.

20. Shermak MA. Pearls and Perils of Caring for the Postbariatric Body Contouring Patient. Plastic and Reconstructive Surgery 2012;130:585e-596e.

21. de Runz A, Brix M, Gisquet H, Pujo J, Minetti C, Colson T, et al. Satisfaction and complications after lower body lift with autologous gluteal augmentation by island fat flap: 55 case series over 3 years. Journal of Plastic, Reconstructive \&
Aesthetic Surgery 2015;68:410-8

22. Kitzinger HB, Cakl T, Wenger R, Hacker S, Aszmann OC, Karle B. Prospective study on complications following a lower body lift after massive weight loss. British Journal of Plastic Surgery 2013;66:231-8.

23. Poodt I, Van Dijk M, Klein S, Hoogbergen M. Complications of Lower Body Lift Surgery in Postbariatric Patients. Plastic and Reconstructive Surgery-Global Open 2016;4:e1030.

24. Kitzinger HB, Abayev S, Pittermann A, Karle B, Kubiena H, Bohdjalian A, et al. The Prevalence of Body Contouring Surgery After Gastric Bypass Surgery. OBES SURG. 2011;22:8-12.

25. Gusenoff JA, Coon D, Rubin JP. Implications of Weight Loss Method in Body Contouring Outcomes. Plastic and Reconstructive Surgery 2009;123:373-6.

26. Zammerilla LL, Zou RH, Dong ZM, Winger DG, Rubin JP, Gusenoff JA. Classifying Severity of Abdominal Contour Deformities after Weight Loss to Aid in Patient Counseling. Plastic and Reconstructive Surgery 2014;134:888e-894e.

27. Hasanbegovic E, Sørensen JA. Complications following body contouring surgery after massive weight loss: A meta-analysis. British Journal of Plastic Surgery. 2014;67:295-301.

28. Constantine RS, Davis KE, Kenkel JM. The Effect of Massive Weight Loss Status, Amount of Weight Loss, and Method of Weight Loss on Body Contouring Outcomes. Aesthet Surg J. 2014;34:57883.

29. Hurwitz D, Aghamohammadi OK, Unadkat J. A Clinical Review of Total Body Lift Surgery. Aesthet Surg J. 2008;28:294-303.

30. Shermak MA, Rotellini-Coltvet LA, Chang D. Seroma Development following Body Contouring Surgery for Massive Weight Loss: Patient Risk Factors and Treatment Strategies. Plastic and Reconstructive Surgery 2008;122:280-8.

31. Buchanan PJ, Nasajpour H, Mast B. Safety and Efficacy of Outpatient Lower Body Lifting. Annals of Plastic Surgery 2013;70:493-6 